Un groupe de travail de l'Association des chefs de service de la Société suisse de médecine interne (SSMI) et de la Société suisse d'endocrinologie et de diabétologie (SSED) a élaboré les recommandations ci-après en vue de l'éducation des patients diabétiques en milieu hospitalier. Le but de ces recommandations est d'optimiser la prise en charge de ces patients au sein des structures hospitalières existantes et d'aboutir à une certaine uniformisation sur le plan national. Ce consensus est également utile à la définition d'un «cadre de prestations» pour la formation postgraduée hospitalière dans ce domaine important de la médecine interne.

Les présentes recommandations, rédigées dans un effort commun par les organismes concernés, démontrent qu'il est possible d'établir en bonne entente des principes utiles en matières de qualité. Dans d'autres domaines également, cet aspect qualitatif de la prise en charge des patients devraient toujours figurer au premier plan. Le Comité de l'Association des chefs de service de la SSMI tient donc à remercier le groupe de travail pour sa prestation exemplaire.

\title{
Recommandations pour une éducation structurée des diabétiques en milieu hospitalier
}

\author{
Association des chefs de service de la Société suisse de médecine interne
}

Deutsch erschienen

in Nr. 35/2002

Correspondance:

Dr Hans-Jürg Vonesch

Bezirksspital

Medizinische Klinik

CH-4800 Zofingen

\section{Objectifs}

Le traitement et l'éducation des patients diabétiques dans les hôpitaux publics font partie des soins de base. La condition préalable est la connaissance des standards modernes de traitement des patients diabétiques (tab. 2).

Chaque hôpital doit, en fonction de ses possibilités, proposer un programme structuré d'éducation des patients. Les médecins-assistants doivent être impliqués dans ce processus. Il convient donc d'élaborer un concept de formation.

\section{Nécessité}

Le diabète sucré est l'affection métabolique la plus fréquente dans le monde et le nombre de cas recensés ne cesse de croître. En Suisse, on dénombre chaque année environ 15000 nouveaux cas, dont les 90\% sont des diabétiques de type 2 . L'importance d'une prise en charge structurée et intensive comprenant le traitement et l'éducation du patient a été attestée par de nombreuses études d'intervention de grande envergure, notamment la DCCT (Diabetes Control and Complications Trial) de 1993 pour les diabétiques de type 1 et la UKPDS (United Kingdom Prospective Diabetes Control Study) de 1998 pour les diabétiques de type 2 . Ces deux études ont permis de démontrer qu'un traitement intensif et complet du diabète, avec une éducation ciblée du patient, permet de réduire les complications macrovasculaires et surtout microvasculaires. Dans la Dé- claration de St-Vincent, placée sous le patronage de l'Organisation mondiale de la santé (OMS) et de la Fédération internationale du diabète (FID), les pays signataires, dont la Suisse, se sont engagés à améliorer la qualité et l'espérance de vie des personnes diabétiques et notamment à réduire le taux d'amputation de $50 \%$ et le taux de rétinopathies et de néphropathies de 30\%. Pour atteindre cet objectif, l'éducation des diabétiques est primordiale et doit faire partie intégrante du traitement. Le fait qu'une éducation intensive a une réelle incidence sur les coûts, à long terme également, a été prouvé dans diverses études, dont celles de Assal.

\section{Organisation}

L'équipe chargée de cette éducation à l'hôpital devrait être composée d'une infirmière en diabétologie, d'une diététicienne et d'un médecin. L'hôpital peut opter pour un personnel spécialisé engagé à plein temps ou à temps partiel ou recourir à des personnes exerçant à titre indépendant, ou encore collaborer avec l'un des centres de consultation de l'Association suisse du diabète. La responsabilité en matière d'éducation et de traitement incombe à la direction médicale. Le médecin-chef peut également confier cette tâche à un médecin-adjoint ou à un chef de clinique. Etant donné que ce dernier n'est en général pas diabétologue, la collaboration, au sens d'un consilium, avec un diabétologue (en cabinet privé ou dans un hôpital disposant d'un service d'endocrinologie et diabétologie) devrait 
Figure 1

Formes d'organisation pour l'éducation et le traitement des diabétiques.

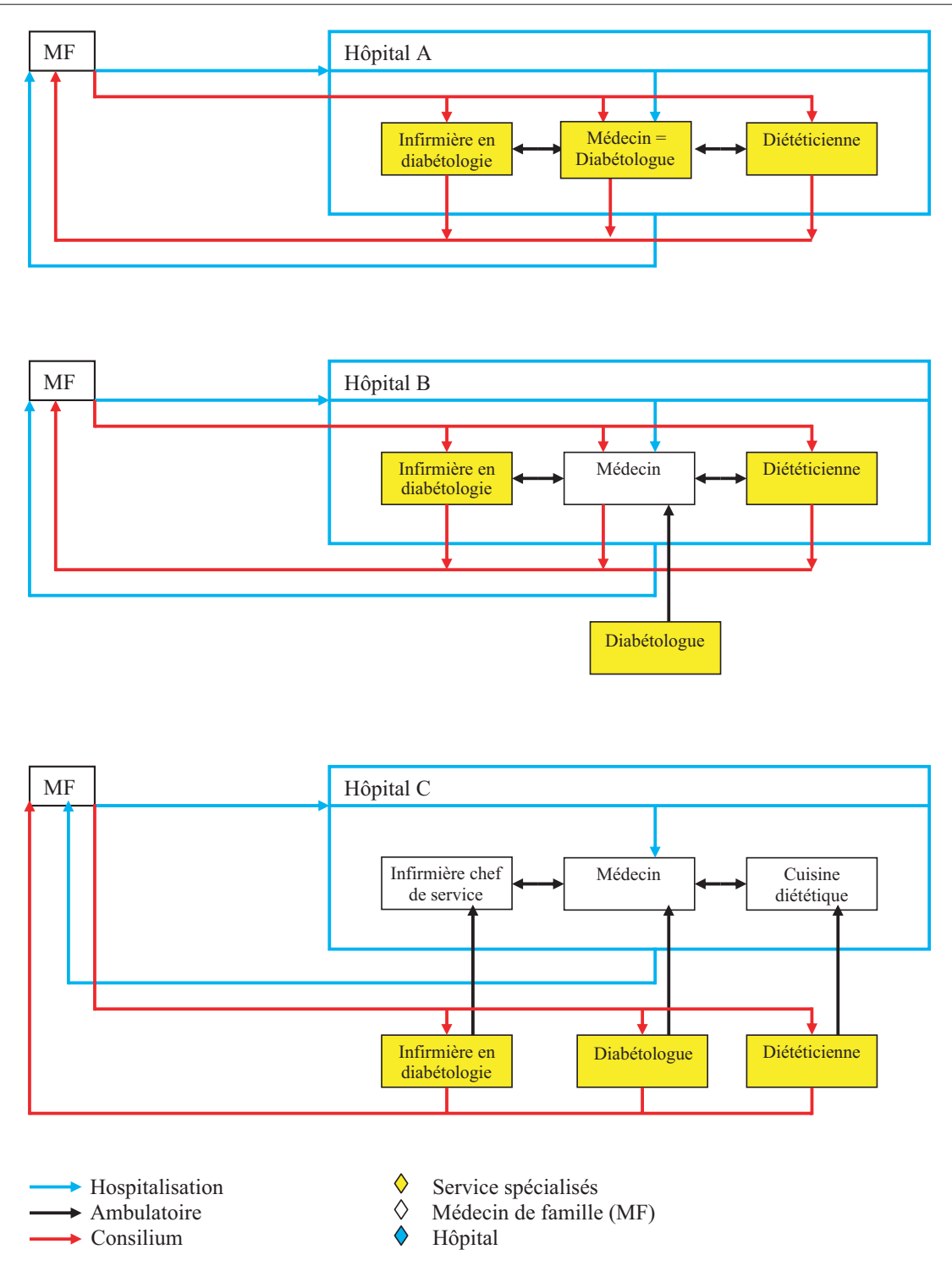

être instituée tant pour la prise en charge des patients que pour la supervision de l'équipe de consultation et pour la formation des assistants (cf. fig. 1 et tab. 1).

Pour la consultation et l'éducation des patients diabétiques ambulatoires et hospitaliers, il convient de mettre à disposition des locaux appropriés ainsi que le matériel d'instruction nécessaire.

Les taux d'occupation de la diététicienne et de l'infirmière en diabétologie sont fixés en fonction des conditions locales. Il arrive fréquemment que l'instruction du patient ne soit pas terminée à la fin du séjour hospitalier et qu'elle doive se poursuivre de manière ambulatoire, d'entente avec le médecin de famille. La plupart des consultations ne peuvent en effet être effec- tuées que de manière ambulatoire. Pour la première consultation-instruction d'un diabétique insulinodépendant, il faut compter 14 à 16 heures et pour un patient non insulinodépendant entre 8 et 12 heures.

\section{Education}

Une éducation structurée et complète du patient diabétique est primordiale pour qu'il puisse atteindre les objectifs de traitement tels qu'ils sont définis aujourd'hui, à savoir: garantir le meilleur équilibre métabolique, le plus d'autonomie et le plus de responsabilité possible en ce qui concerne l'alimentation et l'activité physique, la prise d'antidiabétiques oraux et le traitement à l'insuline, ainsi que l'acceptation du handicap causé par la maladie.

Chaque hôpital devrait, dans la mesure du possible, proposer un programme structuré d'éducation des patients. De nombreux programmes d'excellente qualité sont disponibles en Suisse; ils diffèrent selon la taille et l'infrastructure de l'hôpital et peuvent en outre faire l'objet d'échanges. Au tableau 1, les contenus des programmes d'éducation des patients sont présentés sous forme de modules, dont la séquence et l'étendue doivent être définies individuellement.

\section{Formation continue}

Les infirmières spécialisées en diabétologie et les diététiciennes sont soumises aux directives de leur société ou de leur association professionnelle et les médecins, à celles de la Société suisse d'endocrinologie et de diabétologie (SSED); cf. tab. 2.

Etant donné que les médecins-assistants sont de futurs médecins de premier recours et qu'ils seront appelés à traiter des diabétiques, il est important qu'ils participent à ces programmes d'éducation des patients diabétiques dans les hôpitaux.

Ces recommandations à l'intention des hôpitaux ont été élaborées avec l'accord et le soutien de la Commission médicale et du Groupe de consultation et d'enseignement de l'Association suisse du diabète (ASD), ainsi que du Comité de la SSED.

\section{Groupe de travail de l'Association des chefs de service}

Prof. S. Fankhauser, Starrkirch-Wil;

Dr T. Nanzer, Viège; Dr H. J. Vonesch, Zofingen; Dr D. Wegmann, Stans 


\section{Tableau 1}

Contenu du programme d'éducation des diabétiques.

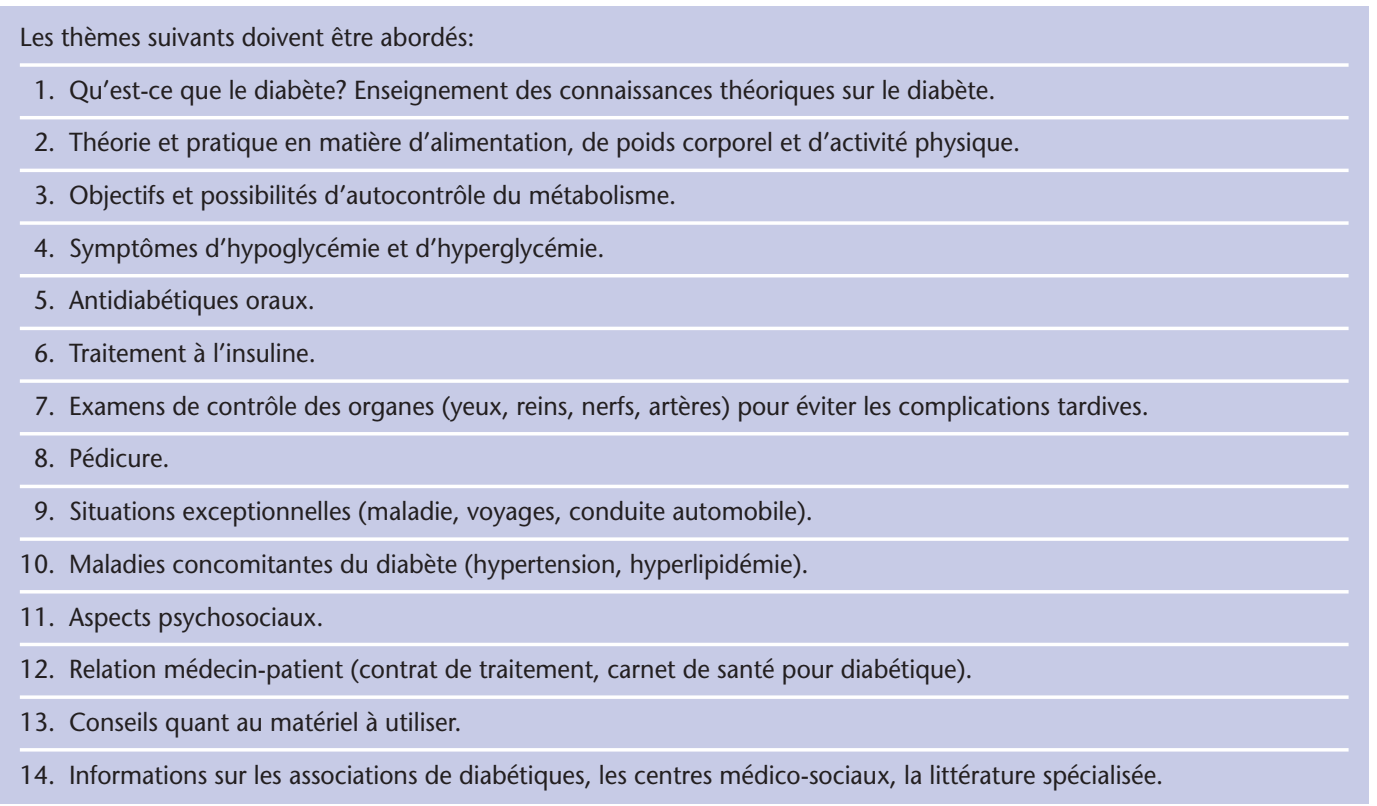

\section{Tableau 2}

Objectifs du traitement du diabète sucré de type 1.

Conformément aux recommandations de la Société américaine de diabétologie émises à l'issue de l'étude DDCT, il faut viser une valeur $\mathrm{HbA} 1 \mathrm{c}$ inférieure à $7 \%$, pour une limite supérieure des sujets normaux de $6 \%$. Il est fréquent qu'au cours des 5 à 10 premières années, on atteigne des valeurs situées entre $6 \%$ et $6,5 \%$ en présence d'une fonction insulinique résiduelle, sans risques d'hypoglycémie sévère.

Le meilleur moyen pour réaliser cet objectif métabolique consiste à éduquer les patients diabétiques de manière intensive en les responsabilisant quant à leur traitement (empowerment).

Objectifs d'un traitement complet du diabète sucré de type 2.

\section{Glycémie}

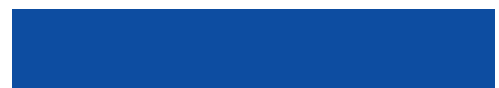

Glycémie à jeun

sang complet capillaire**

Glycémie postprandiale

( $2 \mathrm{~h}$ après un repas)

\section{$\mathrm{HbA1}^{* * *}$}

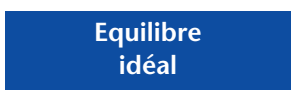

5,0 à $7,0 \mathrm{mmol} / \mathrm{I}$

$$
<8,0 \mathrm{mmol} / \mathrm{l}
$$

5,0 à $7,0 \%$ ou $<0,5 \%$ au-dessus de la norme

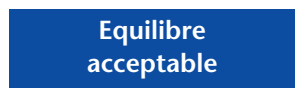

$<8,0 \mathrm{mmol} / \mathrm{l}$

$<10,0 \mathrm{mmol} / \mathrm{l}$

7,0 à $8,0 \%$ ou $<1,5 \%$ au-dessus de la norme

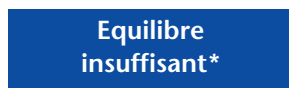

$>8,0 \mathrm{mmol} / \mathrm{l}$

$>10,0 \mathrm{mmol} / \mathrm{l}$

$>8,0 \%$ ou $>1,5 \%$ au-dessus de la norme

* nécessité d'adapter le traitement ** valeurs de $15 \%$ plus élevées dans le plasma *** domaine de référence: 4,0-6,1\%.

- L'autocontrôle de la glycémie est recommandé pour la plupart des diabétiques de type 2, avec définition individuelle du moment et de la fréquence.

- Les valeurs-cibles de glycémie sont à définir pour chaque patient sur la base de l'évaluation globale et du plan thérapeutique (des valeurs augmentées d'environ $1 \mathrm{mmol} / \mathrm{l}$ sont acceptables chez les personnes âgées).

- Les résultats sont discutés avec le patient et consignés dans le carnet de santé pour diabétique. 
Tension artérielle

\section{Valeur idéale}

$<135 / 85$
Valeur acceptable**

$<140 / 90$
Valeur insuffisante*

$>140 / 90$

* nécessité d'adapter le traitement ** chez les patients âgés, des valeurs plus élevées peuvent être adéquates.

- Si au dosage usuel, une substance unique ne permet pas d'obtenir la tension idéale, I'association de plusieurs médicaments est nécessaire pour atteindre l'objectif thérapeutique.

- L'association de plusieurs médicaments faiblement dosés est préférable à une monothérapie fortement dosée.

- Les valeurs-cibles doivent être définies pour chaque patient sur la base de l'évaluation globale et du plan thérapeutique.

- Les résultats sont discutés avec le patient et consignés dans le carnet de santé pour diabétique.

Lipides

Cholestérol total $(\mathrm{mmol} / \mathrm{l})$

$<5,0$
Cholestérol total / HDL

$<5,0$
Cholestérol LDL* $(\mathrm{mmol} / \mathrm{l})$

$<3,0$

* selon la formule de Friedewald, si le taux de triglycérides est $<4,5 \mathrm{mmol} / \mathrm{l}$ : cholestérol $\mathrm{LDL}=$ cholestérol total cholestérol HDL - (triglycérides: 2,2)

Il faut procéder à la vérification d'un traitement médicamenteux lorsque 2 des 3 paramètres lipidiques sont audessus de ces valeurs limites.

- Déterminer 2 ou 3 valeurs lipidiques avant le début d'un traitement médicamenteux.

- S'entretenir des résultats avec le patient et les consigner dans le carnet de santé pour diabétique.

- Contrôle annuel après réalisation de l'objectif thérapeutique.

Prévention des complications tardives

Complications microvasculaires

Rétinopathie

Examen de contrôle du fond de l'œil par l'ophtalmologue au moment du diagnostic. Prévoir des contrôles réguliers par la suite.

Néphropathie

- Test de dépistage de microalbuminurie par spot urinaire matinal (y compris rapport albumine/créatinine).

- Négatif: contrôle 1 fois par an.

- Positif: nécessité d'un contrôle optimal de la tension artérielle. Urine de 24 heures: clairance albumine/créatinine.

Neuropathie (pieds)

- Sensibilité profonde: vibrations, sens de la position, réflexes.

- Sensibilité de surface: monofilaments.

Pieds

- En I'absence de complications neurologiques ou vasculaires: examen 1 fois par an - instructions pour la pédicure.

- En présence de complications neurologiques ou vasculaires (lors de chaque consultation): examens de dépistage des déformations, hyperkératoses, ulcères, infections et mycoses, etc.

- Contrôle des chaussures.

Complications macrovasculaires

- Contrôle du pouls périphérique 1 fois par an / auscultation vasculaire.

- Recherche de cardiopathie coronarienne (N.B.: cardiopathie oligosymptomatique).

Poids

En cas de surcharge pondérale: toute perte de poids, si petite soit-elle, améliore la situation métabolique.

Agrégation des thrombocytes

Il faudrait toujours se demander si une inhibition de l'agrégation des thrombocytes est indiquée.

Points-clés de l'alimentation du diabétique

- Répartition des hydrates de carbone sur 3 repas et 2 à 3 collations.

- Apport suffisant d'hydrates de carbones à chaque repas: éviter les sucres raffinés et leur préférer des hydrates de carbones complexes à absorption lente (fibres) ainsi que des légumes et des fruits ( 2 à 3 fois par jour).

- Consommation de produits laitiers 2 à 3 fois par jour.

- Limitation de la consommation de graisses animales («le gras fait grossir»).

- Consommation régulière de légumes et de salades.

- Limitation de la consommation d'alcool, notamment en cas de surcharge pondérale.

Activité physique

En fonction des possibilités physiques du patient (30 min par jour).

Arrêter de fumer 
Tableau 3

Adresses des centres de consultation.

Association Suisse du Diabète

Forchstrasse 95, 8032 Zurich, tél. 0138313 15, fax 014228912

Association Fribourgeoise du Diabète

Route des Daillettes 1, 1709 Fribourg, tél. 02642602 80, fax 0264260288

Association Genevoise des Diabétiques

Rue de la Synagogue 41, 1204 Genève, tél. 02232917 77, fax 0223291778

Association jurasienne des diabétiques

Case postale 50, 2800 Delémont 1, tél. 03242262 28, fax 0324226228

Association des diabétiques du Jura bernois

Rue Neuve 52, 2613 Villeret, tél. 03294141 21, fax 0329414121

Association Neuchâteloise du Diabète

Rue de la Paix 75, 2301 La Chaux-de-Fonds, tél. 03291313 55, fax 0329131355

Association Valaisanne du Diabète

Rue des Condemines 14, 1950 Sion, tél. 02732299 72, fax 0273229973

Association Vaudoise du Diabète

Chemin de Roveréréaz 5, 1012 Lausanne, tél. 02165719 20, fax 0216571921

Associazione ticinese per i diabetici

Via Motto di Mornera 4, 6500 Bellinzona, tél. 09182595 66, fax 0918263448

Aargauer Diabetes-Gesellschaft

Kantonsspital, Haus 16, 5000 Aarau, Tel. 06282472 01, Fax 0628247258

Diabetes-Gesellschaft Region Basel

Mittlere Strasse 35, 4056 Basel, Tel. 06126103 87, Fax 0612610443

Berner Diabetes-Gesellschaft

Falkenplatz 1, 3012 Bern, Tel. 03130245 46, fax 0313025660

Diabetes-Gesellschaft, GL-GR-FL

Stadtgartenweg 10, 7001 Chur, Tel. 08125350 40, Fax 0812535740

Oberwalliser Diabetes-Gesellschaft

Bachhalteweg 9, 3900 Brig, Tel. 02792435 86, Fax 0279243678

Ostschweizerische Diabetes-Gesellschaft

Neugasse 55, 9000 St. Gallen, Tel. 07122367 67, Fax 0712235892

Diabetes-Gesellschaft des Kantons Schaffhausen

Fronwagplatz 3, 8201 Schaffhausen, Tel. 05262501 45, Fax 0526250146

Solothurner Diabetes-Gesellschaft

Louis-Giroud-Strasse 20, 4601 Olten, Tel. 06229680 82, Fax 0622968082

Zentralschweizerische Diabetes-Gesellschaft

Würzenbachstrasse 54, 6006 Luzern, Tel. 04137031 32, Fax 0413703138

Diabetes-Gesellschaft Zug

Artherstrasse 25, 6300 Zug, Tel. 04170987 69, Fax 0417097838

Zürcher Diabetes-Gesellschaft

Hegarstrasse 18, 8032 Zürich, Tel. 0138300 60, Fax 013830692 